\title{
Laser-Combined Scanning Tunneling Microscopy on the Carrier Dynamics in Low-Temperature-Grown GaAs/AlGaAs/GaAs
}

\author{
Yasuhiko Terada, Shoji Yoshida, Osamu Takeuchi, and Hidemi Shigekawa
}

Institute of Applied Physics, University of Tsukuba, Tsukuba 307-8573, Japan

Correspondence should be addressed to Hidemi Shigekawa, hidemi@ims.tsukuba.ac.jp

Received 31 May 2011; Accepted 26 August 2011

Academic Editor: Qingming Luo

Copyright (๑) 2011 Yasuhiko Terada et al. This is an open access article distributed under the Creative Commons Attribution License, which permits unrestricted use, distribution, and reproduction in any medium, provided the original work is properly cited.

\begin{abstract}
We investigated carrier recombination dynamics in a low-temperature-grown GaAs (LT-GaAs)/AlGaAs/GaAs heterostructure by laser-combined scanning tunneling microscopy, shaken-pulse-pair-excited STM (SPPX-STM). With the AlGaAs interlayer as a barrier against the flow of photocarriers, recombination lifetimes in LT-GaAs of 4.0 ps and GaAs of 4.8 ns were successfully observed separately. We directly demonstrated the high temporal resolution of SPPX-STM by showing the recombination lifetime of carriers in LT-GaAs (4.0 ps) in the range of subpicosecond temporal resolution. In the carrier-lifetime-mapping measurement, a blurring of recombination lifetime up to $50 \mathrm{~nm}$ was observed at the LT-GaAs/AlGaAs boundary, which was discussed in consideration of the screening length of the electric field from the STM probe. The effect of the built-in potential on the signal, caused by the existence of LT-GaAs/AlGaAs/GaAs boundaries, was discussed in detail.
\end{abstract}

\section{Introduction}

The development of semiconductor physics and devices has been progressing with the innovation of atomically controlled fabrication technologies [1-3]. However, the carrier dynamics in materials have been analyzed only by evaluating macroscopic functions, or with techniques that provide spatially and/or temporally averaged information. Microscopic carrier dynamics has not been directly observed, although it is fundamental for the device functions. Therefore, there has been an increasing desire for developing a microscopic technique that allows the investigation of carrier dynamics at high spatial and temporal resolutions.

Laser-combined scanning tunneling microscopy (STM) is a promising candidate, because the tunneling junction itself responds on a time scale of a few femtoseconds and thereby ultrafast dynamics can principally be studied by STM with ultrashort pulse laser techniques [4-12]. Recently, we have reported a novel microscopy technique that enables the visualization of subpicosecond carrier dynamics in nanometer-scale structures [13], that is, shakenpulse-pair-excited scanning tunneling microscopy (SPPXSTM) with a novel delay-time modulation method based on a pulse-picking technique [14-21]. A nonequilibrium carrier distribution is generated with ultrashort laser pulses and its relaxation processes are probed using STM by a pump-probe technique. We proposed a model clarifying the physical origin of the signal for semiconductors and verified it experimentally. The SPPX-STM signal consists of two components, that is, a faster one reflecting the decay process of bulk-side photocarriers and a slower one reflecting the decay process of surface-side photocarriers. Bulk-side carrier lifetimes in various semiconductor materials were successfully measured using tunneling current, and the hole capture rate by the gap states formed by the nanometer-scale Co islands on GaAs was directly obtained with an atomic spatial resolution through the analysis of the surface-side carrier dynamics.

In this paper, we focus on the SPPX-STM measurement on the bulk-side carrier dynamics in a nanostructure consisting of materials with different recombination lifetimes, namely, a low-temperature-grown GaAs (LTGaAs)/AlGaAs/GaAs heterostructure. The factors determining the spatial resolution of SPPX-STM for the measurement of bulk-side decay are discussed in detail. 


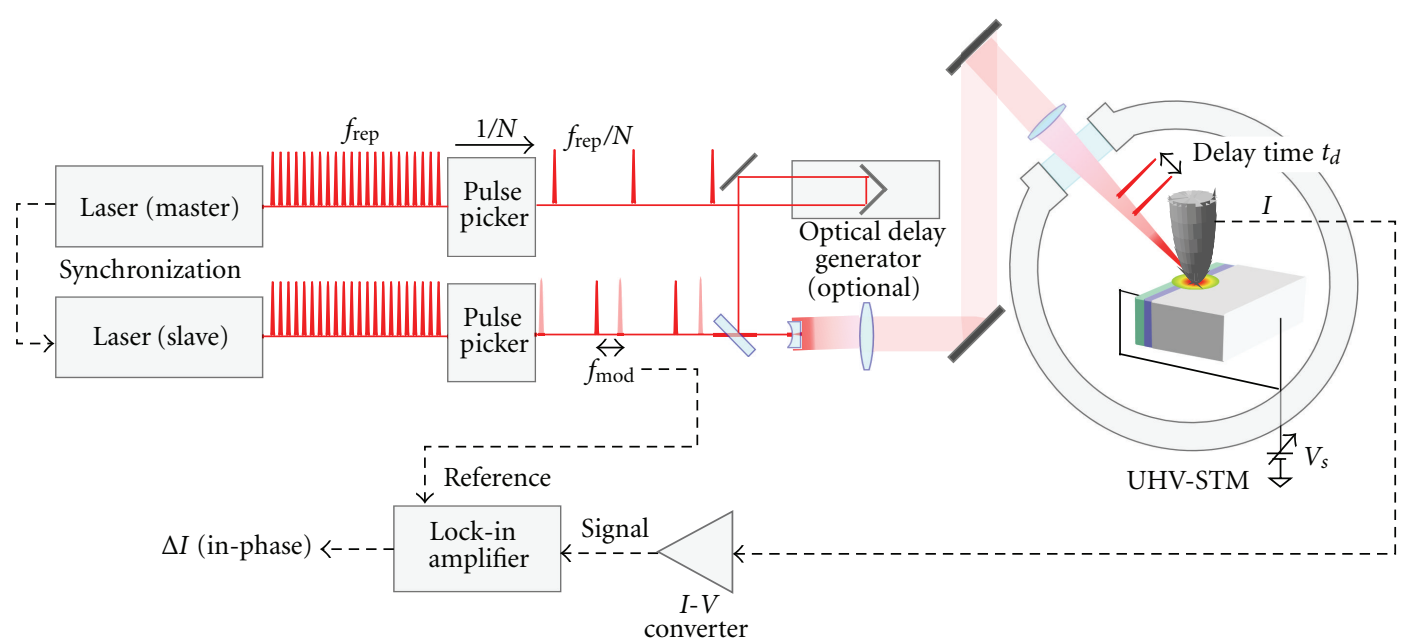

FIGURE 1: Schematic of SPPX-STM.

\section{Experimental Methods}

2.1. SPPX-STM Measurement. Figure 1 shows a schematic of the SPPX-STM system with the pulse-picking technique, the details of which are described elsewhere [13]. In short, each of two (pump and probe) pulse trains generated by two synchronized Ti:sapphire lasers (Mira and Chameleon, Coherent Inc, central wavelength $=800 \mathrm{~nm}$, repetition rate $=$ $90 \mathrm{MHz}$, pulse width $=140 \mathrm{fs}$ ) was guided to a pulse picker, which consisted of an ultrafast Pockels cell and polarizers. The delay time between the two pulse trains was continuously varied from zero to the pulse interval ( $\sim 11 \mathrm{~ns})$ by the synchronizing circuit (Synchrolock, Coherent Inc.), or varied from zero to $1 \mathrm{~ns}$ by an ordinary optical delay line. The pulse picker selectively transmitted typically one pulse per every 90 pulses, resulting in a pulse train with a reduced repetition rate of $f_{p}$ (typically $1 \mathrm{MHz}$ ). Then, the two pulse trains were arranged onto the same optical axis and guided into the STM (VT-STM, Omicron Nanotechnology GmbH). The light spot, which had a diameter of less than $10 \mu \mathrm{m}$ and an average intensity of up to several milliwatts, was focused on a sample surface below the STM tip using lenses placed outside the UHV chamber (base pressure $<1 \times 10^{-8} \mathrm{~Pa}$ ). All the experiments discussed in this paper were carried out at room temperature. In most cases, SPPX-STM signal is so weak that lock-in detection with a modulation technique is required for observation. In the conventional optical pump-probe experiments, the laser-intensity modulation is generally used, but this is not directly applicable to SPPXSTM because it produces thermal expansion and shrinking of the STM tip in synchronization with modulation, and consequently results in a large change in tunneling current. A promising way to overcome this difficulty is to modulate delay time instead of laser intensity. Delay time modulation was achieved by changing the pulse-picking timing periodically at a frequency of $\sim 1 \mathrm{kHz}$. The delay time was modulated in a rectangular form between a relatively small value $t_{d}(1 \mathrm{ps}-1 \mu \mathrm{s})$ and a very large value $(0.5 \mu \mathrm{s}-$ several $\mu \mathrm{s})$, by which the ultrafast phenomena reached the steady state. Synchronously with the delay time modulation, the tunneling current signal from the STM preamplifier with a bandwidth of $\sim 10 \mathrm{kHz}$ was phase-sensitively detected using a lock-in amplifier. By virtue of the rectangular modulation of the delay time, the output of the lock-in amplifier was proportional to the difference between the tunneling current at the two delay times. In particular, when the larger delay time was set to be sufficiently long for transient phenomena to reach the steady state, the signal can be regarded as proportional to $\Delta I=I\left(t_{d}\right)-I(\infty)$. The sign of $t_{d}$ was defined to be positive when the pump pulse illuminated the sample first. Unlike the optical pump-probe technique, here, pump and probe pulses were adjusted to have the same intensity to produce a symmetric $\Delta I$ curve with respect to the origin [17].

2.2. LM-STS Measurement. Light-modulated scanning tunneling spectroscopy (LM-STS) was used for analyzing the band structure of the sample [19, 21-25]. In LM-STM measurement, the sample under STM is intermittently illuminated by an electrically chopped laser source and the sample bias voltage is swept to obtain tunneling current versus sample bias voltage $\left(I-V_{s}\right)$ curves underilluminated and dark conditions simultaneously, which provides information for analyzing the band structure of the sample. In STM on a semiconductor sample in the dark condition, the bias voltage applied between the tip and the sample penetrates into the sample within the screening length and causes, so called, tipinduced band bending (TIBB). When the sample is photoilluminated, the redistribution of photocarriers reduces the band bending, that is, surface photovoltage (SPV). The amount of SPV, thus TIBB, is estimated from the shift of the two $I-V_{s}$ curves, providing information about the carrier density due to doping in the dark condition.

2.3. Sample Preparation. A layer of LT-GaAs $(1 \mu \mathrm{m})$ was grown at $250^{\circ} \mathrm{C}$ by molecular beam epitaxy on a barrier layer of $\mathrm{Al}_{x} \mathrm{Ga}_{1-x} \mathrm{As}(x=0.5)(1 \mu \mathrm{m})$ grown on an undoped 
GaAs substrate, which was annealed at $700^{\circ} \mathrm{C}$ for $60 \mathrm{~s}$ in $\mathrm{H}_{2}$ (5\%)/Ar ambient. The energy bandgaps are $1.43 \mathrm{eV}$ for GaAs and LT-GaAs and $1.97 \mathrm{eV}$ for AlGaAs. The excitation photon energy was $1.55 \mathrm{eV}$ (wavelength $=800 \mathrm{~nm}$ ), and thus, no photocarriers are generated in AlGaAs. The heterojunctions were type I, and the AlGaAs interlayer is expected to act as a barrier that prevents photocarriers generated in the adjacent LT-GaAs and GaAs layers from mixing with each other. The sample was cleaved in ultrahigh vacuum (UHV), and the cleaved surface, including GaAs/AlGaAs and AlGaAs/LTGaAs interfaces, was used for measurements. The sample was clamped by a metallic holder, and thereby, the sample bias voltage was applied through both the ends of the layered structure as shown in Figures 1 and 3.

\section{Results and Discussion}

3.1. Band Structure Investigated by LM-STS. Figure 2 shows an STM image of the cleaved surface. The LT-GaAs/AlGaAs and AlGaAs/GaAs interfaces, which extend in the longitudinal direction in this figure, are clearly distinguishable and they were used to determine the tip location. Then, we performed LM-STS measurement to investigate the band structure of the sample (Figure 3). As described later, its detailed understanding is essential for interpreting the timeresolved signals $(\Delta I)$ obtained by SPPX-STM. For example, not only the local potential below the STM tip but also the distribution of the potential over the sample, such as an inner potential, influences the result of SPPX-STM measurement, and LM-STM is useful to discriminate such effects from each other.

As described in Section 2.2, $I-V$ curves under illuminated and dark conditions are simultaneously obtained by LMSTM measurement, which provides the information for analyzing the band structure of the sample. First, the $I-V_{s}$ curve in the dark for GaAs (blue line in Figure 3(b)) reveals that the tunneling current does not flow unless a bias voltage much larger than the bandgap energy is applied. This is because large TIBB occurs at both positive and negative bias voltages $[20,22-25]$. This tendency is also present for AlGaAs and LT-GaAs (Figures 3(c) and 3(d)). These results indicate that the impurity density is extremely low for all the layers and the materials are semi-insulating, which is reasonable because the sample is undoped. Meanwhile, although the onset voltage is $2.5 \mathrm{~V}$ for $V_{s}>0$ for all regions, for $V_{s}<$ 0 the onset voltage is $-2.5 \mathrm{~V}$ for GaAs, whereas it is not observed at $V_{s}>-3 \mathrm{~V}$ for both AlGaAs and LT-GaAs. This indicates that for AlGaAs and LT-GaAs, the electron density is extremely low compared with the hole density, and consequently, these layers exhibit a p-type-like feature although they are undoped.

Second, the $I-V_{s}$ curves show that a larger current flows under the illuminated condition for GaAs and LT-GaAs (red lines in Figures 3(b) and 3(d)) than under the dark condition (especially for $V_{s}<0$ ). This is because the band, which is bent in the dark owing to TIBB, relaxes towards the flatband state, resulting in the generation of SPV [23, 25-28]. The change in current is larger for $V_{s}<0$ than for $V_{s}>0$.

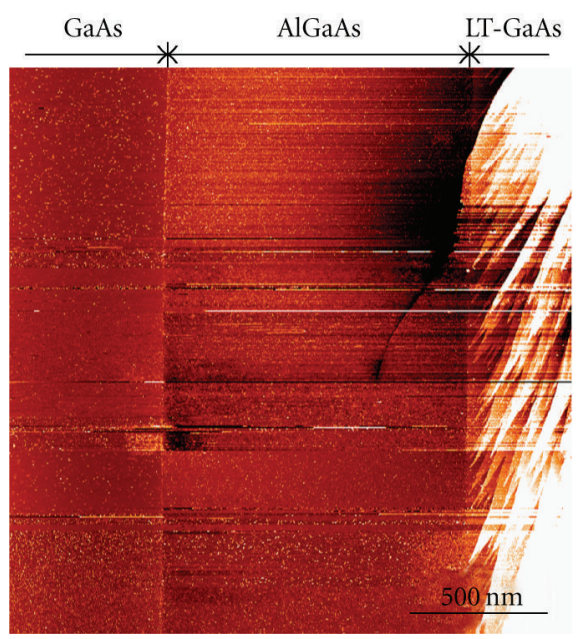

Figure 2: STM image of cleaved surface of GaAs/AlGaAs/LT-GaAs sample. $V_{s}=5.5 \mathrm{~V}, I=45 \mathrm{pA}$.

This also ensures that the GaAs and LT-GaAs layers are ptype-like $[29,30]$. Here, however, the change in current due to photo illumination is also observed at $V_{s}>0$, which is not supposed to occur for intentionally p-doped samples because negligible band bending is produced for the forward bias condition. In the present case, the density of majority carriers (hole) is quite low as described above. Thus, TIBB and the resulting SPV are noticeable even under a forward bias condition.

In contrast, for AlGaAs, the tunneling current under the illuminated condition is almost the same as that in the dark (Figure 3(c)). This indicates that no photocarriers are generated and the AlGaAs layer works well as a barrier against the flow of photocarriers from the adjacent LT-GaAs and GaAs layers, as expected. If photocarriers had flowed from GaAs or LT-GaAs into AlGaAs, TIBB in AlGaAs would have been reduced, as observed at the pn junction of GaAs in previous studies [23-25]. The slight changes seen in the red and blue curves in Figure 3(c) are due to the effect of photovoltage at the LT-GaAs/AlGaAs/GaAs interfaces, which will be discussed in Section 3.4.

3.2. Time-Resolved Signals for Each Region. Figure 4 shows time-resolved signals obtained in each region. The $\Delta I\left(t_{d}\right)$ curve for GaAs (Figure 4(a)) exhibits an exponential-decay component with the time constant of $4.8 \mathrm{~ns}$. The origin of the time-resolved signals is discussed in detail in [13]. In short, for nearly semi-insulating samples, TIBB occurs in the dark at both positive and negative bias voltages. Figure 5(a) shows the band diagram in the depth direction under the positively biased condition. As the sample is illuminated by the optical pulse, photogenerated minority carriers are redistributed immediately, TIBB vanishes, and the band becomes flat, resulting in an increase in the tunneling current (Figure 5(b)). This is followed by carrier decay in the bulk side, where the band still remains flat (Figure 5(c)). The $\Delta I\left(t_{d}\right)$ signals shown in Figure 4 reflect the bulk-side decay process through the absorption bleaching mechanism 


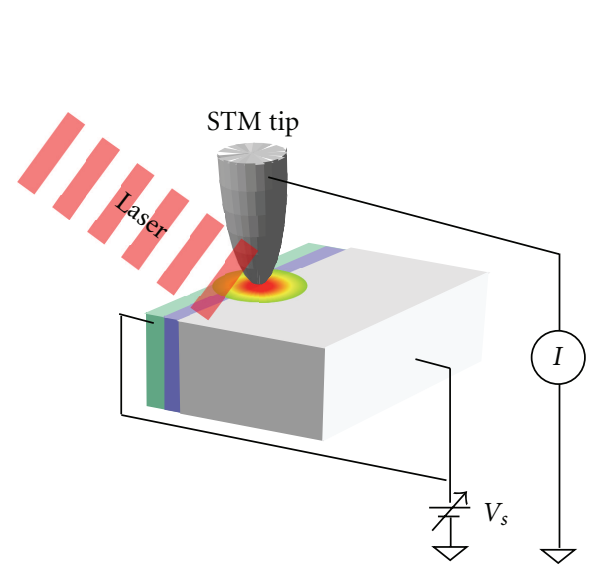

(a)

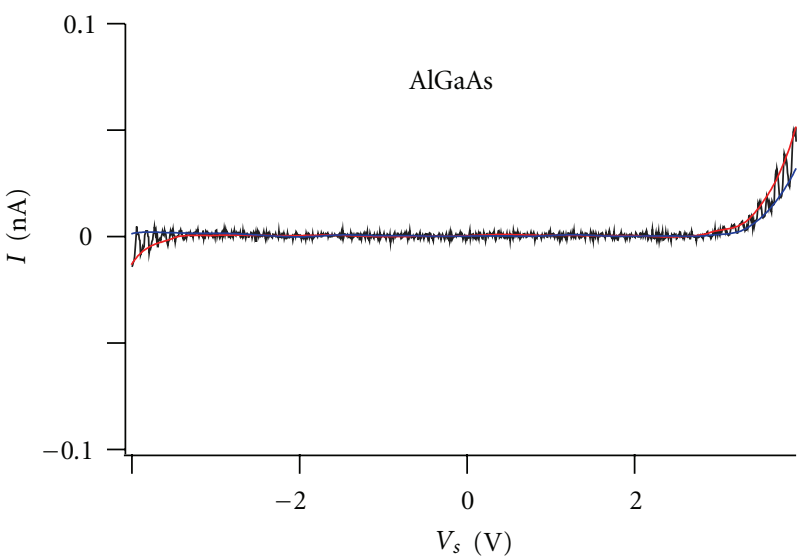

(c)

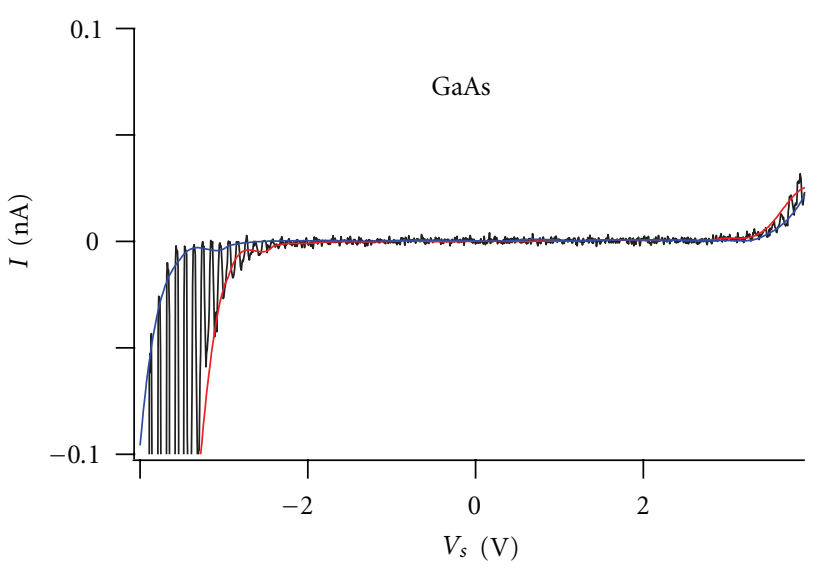

(b)

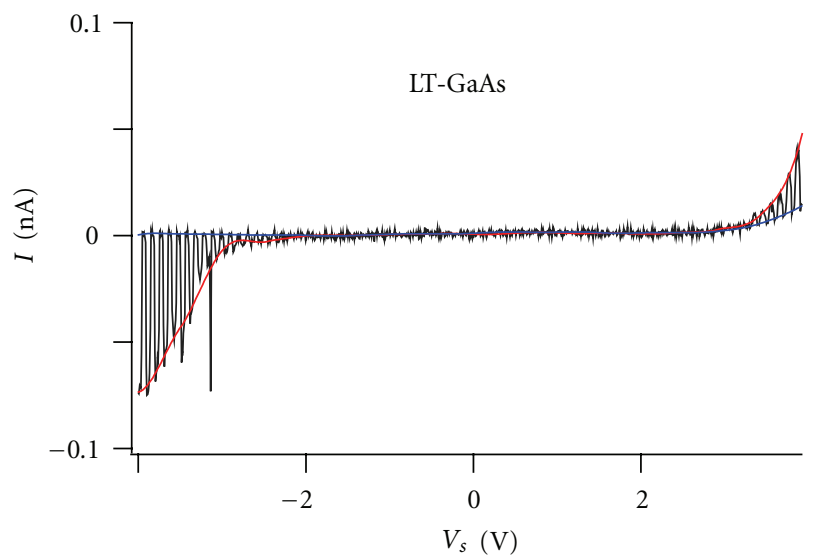

(d)

FIGURE 3: LM-STS measurement and results. (a) Schematic of measurement setup. (b)-(d) $I$ - $V_{s}$ spectra obtained for (b) GaAs (200 nm from the GaAs/AlGaAs interface), (c) AlGaAs (200 nm from the GaAs/AlGaAs interface), and (d) LT-GaAs (150 nm from the AlGaAs/LT-GaAs interface). Red and blue curves correspond to the curves with and without illumination, respectively. Oscillation between the two curves is due to the illumination being switched on and off at $100 \mathrm{~Hz} . V_{s}=4 \mathrm{~V}, I=50 \mathrm{pA}$.

$[31,32]$. A long time later, the surface-side carriers decay (Figure 5(d)) and the band relaxes to the original state. This process is also visible in SPPX-STM measurement; however, since it is a much slower process than the bulk-side carrier decay, it appears merely as an offset within the short delaytime range, as shown in Figure 4.

The sign of $\Delta I\left(t_{d}\right)$ is negative, because the probing process is based on the mechanism of absorption bleaching. The reduction in current gives a measure of the photocarrier density remaining at the illumination of the second pulse. As shown in Figures 4(a) and 4(c), the bulk-side carrier decay time is $4.0 \mathrm{ps}$ for LT-GaAs, which is much shorter than that for GaAs (4.8 ns). This is interpreted as follows: LT-GaAs contains dense defects that act as sites for carrier recombination $[31,32]$ and thus has an ultrashort carrier lifetime appearing in $\Delta I[33,34]$. The decay constants are consistent with the recombination lifetimes obtained by the conventional optical pump-probe technique [13].

The result indicates that AlGaAs works well as a barrier layer. Otherwise, part of the photocarriers in GaAs may flow to the LT-GaAs layer. SPPX-STM probes not the recombination rate itself but the temporal and spatial changes in the carrier density. Thus, it is important to know and manipulate the band structure of the sample to maximize the advantageousness of the SPPX-STM measurement.

3.3. Lifetime Mapping. To examine the possibility of lifetime mapping, we measured line profiles of $\Delta I$ across the LTGaAs/AlGaAs interface by slowly scanning the STM tip $(\sim 10 \mathrm{~nm} / \mathrm{s})$ with the delay time fixed (Figure 6(a)). The profiles are presented with $0.5 \mathrm{pA}$ steps for clarity. First, $\Delta I$ at the longest delay $\left(t_{d}=+29 \mathrm{ps}\right)$ shows an offset, which is attributed to the decay component with a long lifetime shown in Figure 4. Second, for shorter delay times, $\Delta I$ in AlGaAs shows almost the same value independently of delay time, while $\Delta I$ in LT-GaAs markedly decreases as the delay time decreases, especially at delay times from +0.3 to $+4.6 \mathrm{ps}$. The change in $\Delta I$ for LT-GaAs on an ultrashort picosecond timescale is attributed to the change in the density of bulk-side carriers. No ultrafast response is observed for AlGaAs because no photocarriers are generated as described in Section 3.1. The reason why $\Delta I$ for AlGaAs has the offset will be described in Section 3.4. In this way, the microscopy technique has an advantage in visualizing carrier 


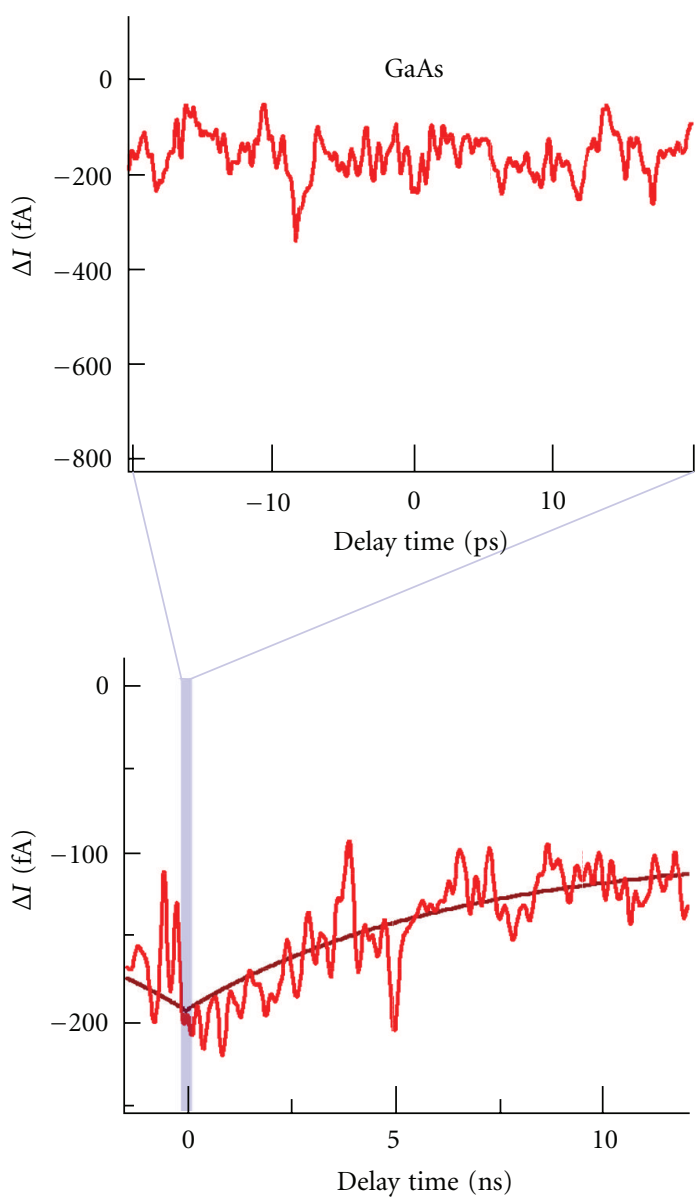

(a)

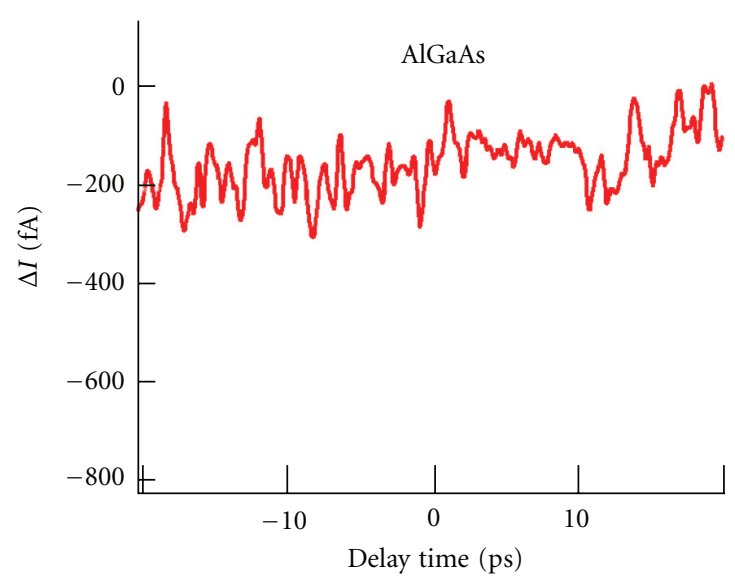

(b)

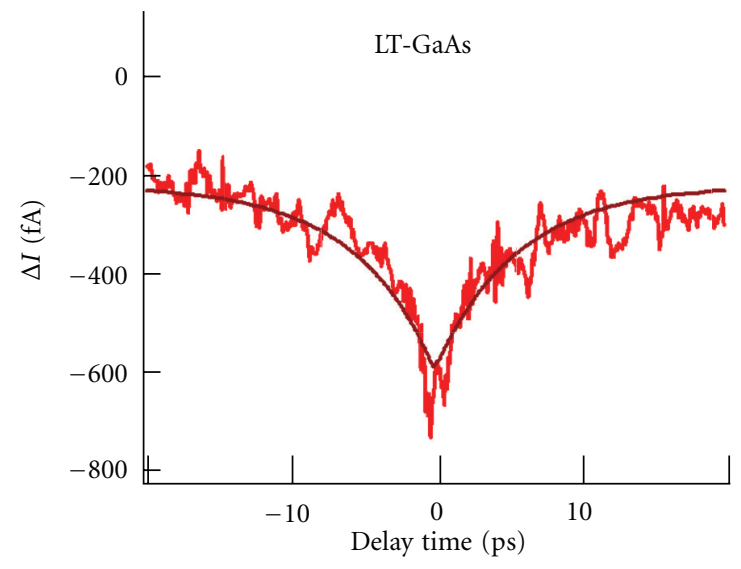

(c)

Figure 4: Results of time-resolved measurement ( $\Delta I$ versus $t_{d}$ curves) for (a) GaAs (400 nm from the GaAs/AlGaAs interface), (b) AlGaAs $\left(180 \mathrm{~nm}\right.$ from the AlGaAs/GaAs interface), and (c) LT-GaAs (75 nm from the AlGaAs/LT-GaAs interface) regions. $V_{s}=5.5 \mathrm{~V}, I=45 \mathrm{pA}$.

dynamics on the ultrasmall scale compared with the optical wavelength.

Next, we focus on a strange phenomenon appearing near the interface. The ultrafast component observed in LT-GaAs is also observed in AlGaAs near the boundary. It spatially decays in the AlGaAs region from the boundary with a decay constant of $\sim 50 \mathrm{~nm}$. Such an ultrafast phenomenon is unlikely to occur in AlGaAs because of no photocarrier generation in this region and no photocarrier inflow from the LT-GaAs region. Therefore, this component is considered to reflect the carrier dynamics in LT-GaAs. In fact, the ultrafast component observed in AlGaAs has the same decay constant as that in LT-GaAs within the experimental error. This indicates the existence of a mechanism that degrades the spatial resolution of recombination lifetime measurement. In the following, we assume that the blurring is caused by the spatial broadening of TIBB and discuss its effect on the measurement.

In the dark, the band bends owing to the electric field from the STM tip. This TIBB broadens in the in-plane direction as well as in the depth direction. Here, let us consider the case in which the STM tip is located in the $\mathrm{AlGaAs}$ region close to the interface. When the sample is positively biased, the band bends upward (Figure 6(b)-(i)). When the sample is illuminated, the photocarriers generated in LT-GaAs are separated by the electric field due to TIBB, and flatten the band in the region (Figure 6(b)-(ii)). As a result, the finite SPV generated beneath the STM tip increases and $\Delta I$ measured at the position becomes sensitive to the carrier decay in the LT-GaAs region. Even when the tip is located in the AlGaAs region, if the electric field beneath the STM tip expands to the LT-GaAs region, $\Delta I$ is affected by the change in TIBB (or SPV) at the LT-GaAs side. This expectation is consistent with the experimental result that the $\Delta I$ in the AlGaAs region near the AlGaAs/GaAs interface has the same ultrashort decay constant as that in LT-GaAs.

Consequently, the spatial resolution of the bulk-side carrier density measured by SPPX-STM is determined by the size of the regions over which TIBB occurs, that is, the screening length of the sample. In the present case, the length is $50 \mathrm{~nm}$. Generally, the screening length is dependent on various factors such as the carrier density. The screening length increases as the doping becomes lower. In the present case, the undoped $\mathrm{AlGaAs}$ has low carrier density, which is not a favorable condition for the spatial measurement. 


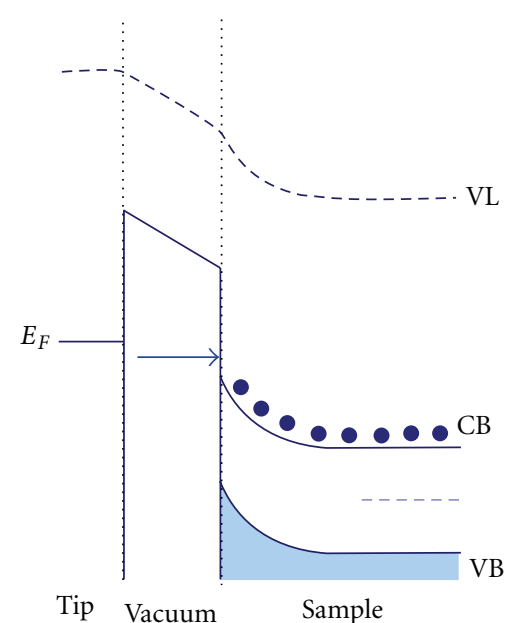

(a)

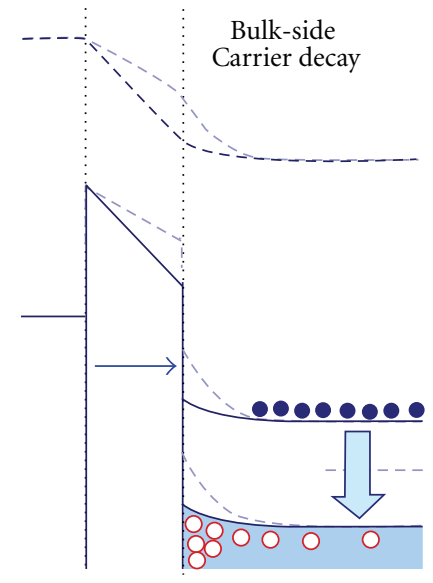

(c)

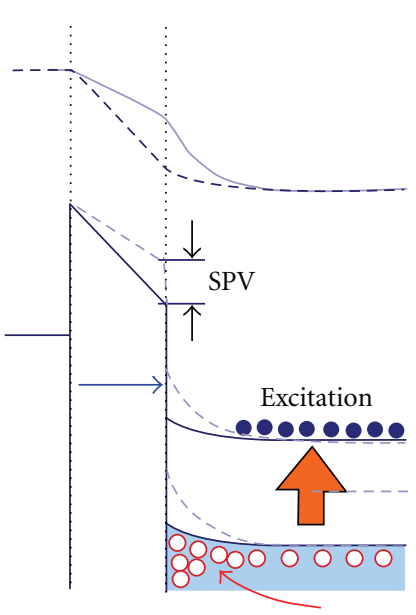

(b)

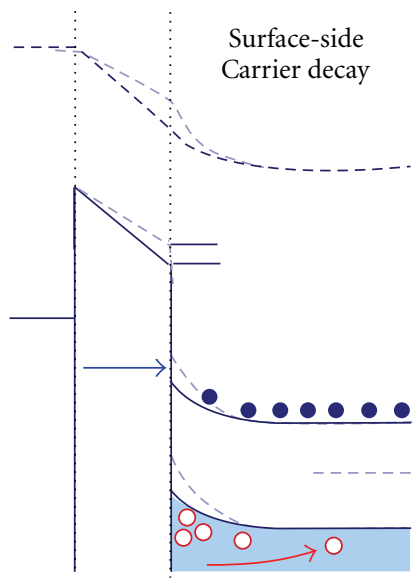

(d)

FIGURE 5: Time evolution of in-depth band diagram (a) before excitation, (b) immediately after excitation, (c) during bulk-side carrier decay, and (d) during surface-side carrier decay. VL, CB, VB, and $E_{F}$ denote the vacuum level, conduction band, valence band, and Fermi level energy, respectively. Blue and red circles are electrons and holes, respectively.

In [13], we measured the carrier capture rate at the atomic resolution. We could achieve such a high resolution because we observed the surface-side carrier decay rather than the bulk-side carrier decay. In that case, the decay process was dominated by the direct recombination of photocarriers and tunneling electrons at the localized state on the surface, and therefore, the spatial resolution of the tunneling current, that is, the atomic resolution, was realized.

3.4. Effect of Built-In Potential. As shown in Figure 6(a), $\Delta I$ at $t_{d}=+29 \mathrm{ps}$ is not zero even in AlGaAs. Moreover, the result shown in Figure 4(b) reveals that the decay time of this component is also long. This slow component is attributed to the carrier dynamics in the built-in potential generated at the LT-GaAs/AlGaAs/GaAs interfaces as discussed below. Figure 7 shows a band diagram in the in-plane direction. Since we did not carry out doping by design for this sample, it is difficult to determine the detailed band structures, for example, the length where the band bends (depletion width) for each layer. Therefore, the depletion width in AlGaAs was determined here in accordance with the result shown in Figure 6(a). The depletion widths for other layers should be longer, but since the exact dimensions are not important here, they were simply drawn to be the same as that for AlGaAs. In addition, the band structure at each interface, which depends on the relation of the Fermi levels in both sides, influences the sign of the time-resolved signal $\Delta I$, as discussed in [13], but does not affect the following discussion on $\Delta I$. The diagram in Figure 7 is drawn in consideration of the IV curves and the sign of the signal observed.

In the dark (Figure 7(a)), holes drift to the AlGaAs/GaAs interface and the area where the acceptors remain is negatively charged. Consequently, the built-in potential is formed at the interface in such a direction that the AlGaAs band is raised. Since the sample is biased on the both ends of the layered sample (Figures 1 and 3), this built-in potential energetically shifts the bands in the AlGaAs [25]. Furthermore, a similar built-in potential is generated at the LT-GaAs/GaAs interface through the same mechanism. 


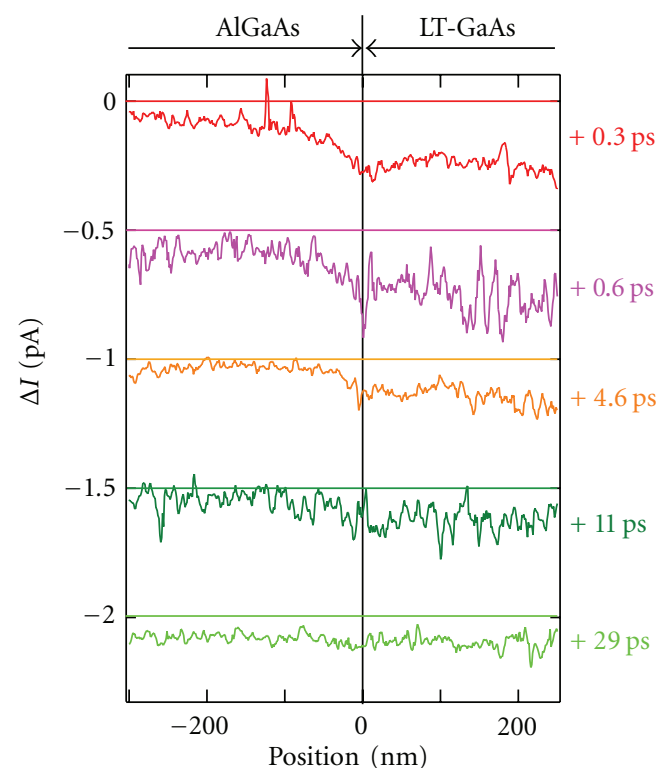

(a)

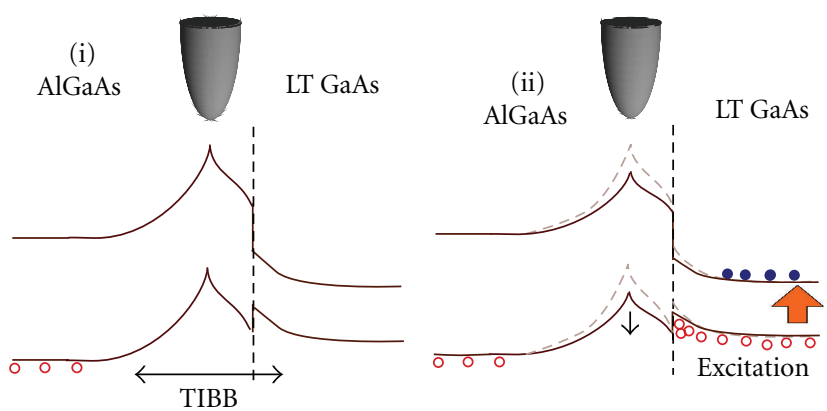

(b)

FIgure 6: (a) Line profiles of $\Delta I$ at different $t_{d}$ with the tip scanned across the AlGaAs/LT-GaAs interface. Each profile is offset by 0.5 pA steps for clarity. (b) Time evolution of in-plane band diagram near the interface: (i) before excitation and (ii) immediately after excitation. $V_{s}=5.5 \mathrm{~V}, I=45 \mathrm{pA}$.

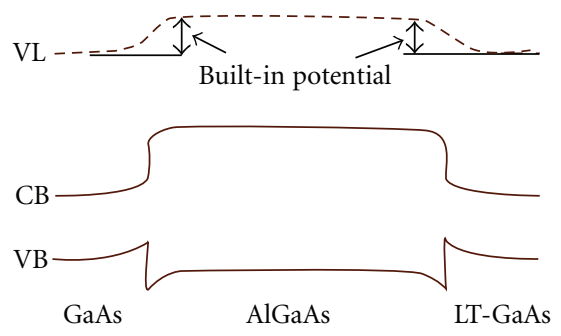

(a)

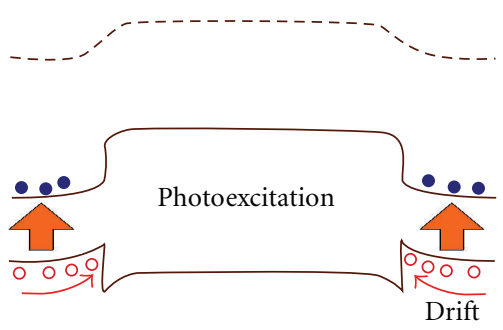

(b)

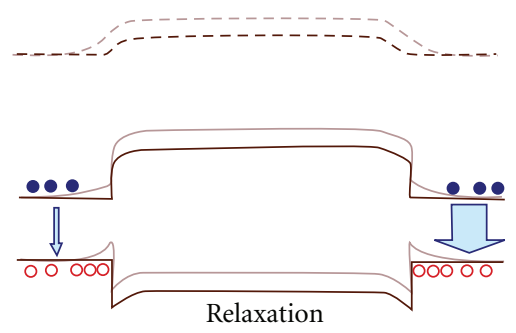

(c)

FIGURE 7: Effect of built-in potential on the band diagram: (a) before excitation, (b) immediately after excitation, (c) relaxation due to the decay of the photocarrier density at the interface (from dark brown lines to light brown lines).

When the sample is illuminated, the laser spot (several micrometers in diameter) covers the entire LT$\mathrm{GaAs} / \mathrm{AlGaAs} / \mathrm{GaAs}$ regions, and thereby, photocarriers are generated in LT-GaAs and GaAs (Figure 7(b)). The LT-GaAs and $\mathrm{GaAs}$ bands become flat owing to the photocarrier drift (Figure 7(c), dark brown lines), and thereby, the AlGaAs band shifts downward because both the ends of the sample are at the same bias voltage as explained in Section 2.3 (Figures 1 and 3). This is the effect of the photovoltages produced at the LT-GaAs/AlGaAs and AlGaAs/GaAs interfaces. In this situation, the vacuum level decreases and the voltage between the tip and sample increases, resulting in an increase in the tunneling current. Then, the change photoinduced at the built-in potential relaxes, as the photocarriers in the LT-GaAs and GaAs regions decay (Figure 7(c), light brown lines).

The above relaxation process of the built-in potential also appears in the time-resolved signal through the change in the tunneling current. The sign of this component changes with the bias voltage. For $V_{s}>0$, the tunneling current increases owing to photocarrier generation, and hence, the current deviation due to absorption bleaching is negative $(\Delta I<0)$. The sign of the signal measured for AlGaAs is consistent with this interpretation.

In the GaAs and LT-GaAs regions, $\Delta I$ reflects the bulkside and surface-side decay processes through the change in TIBB and SPV between the tip and the sample. In contrast, in the AlGaAs region far from the GaAs/AlGaAs and $\mathrm{AlGaAs} / \mathrm{GaAs}$ interfaces, no photocarrier is generated and flows from the adjacent layers, and therefore, $\Delta I$ reflects not the change in TIBB between the tip and the sample but the change in SPV arising from the built-in potential at the two interfaces. When the tip is located in the AlGaAs region near the interfaces and the electric field beneath the tip reaches the GaAs or LT-GaAs region, $\Delta I$ is affected by both the fast and 
slow decay processes in each region. The slow component only appears as the offset component within the ultrashort time range as shown in Figure 4. Since the amounts of the SPVs at the build-in potentials depend on the absorption bleaching processes in the GaAs and LT-GaAs regions, a fast component is, in principle, expected to be observed even in the AlGaAs region as an effect of the build-in potential. The reason for the no observation of such a signal may be explained by the fact, for example, that the ratio of the signal intensities between fast and slow components depends on the laser intensity, the details of which will be discussed elsewhere.

3.5. Temporal Resolution. Here, we discuss the temporal resolution. In the present case, we directly demonstrated the high temporal resolution of SPPX-STM by showing the recombination lifetime of carriers in LT-GaAs (4.0 ps) in the range of subpicosecond temporal resolution (Figure 6(a)). Results of the detailed analysis of the shape of signal $\Delta I\left(t_{d}\right)$, which was discussed in the supplementary information in [13], showed that the temporal resolution is determined by the duration of the excitation laser pulse ( $140 \mathrm{fs}$ in the present case). The introduction of an excitation pulse with a shorter duration such as $10 \mathrm{fs}$ will enable the probing of faster phenomena with higher temporal resolution.

3.6. Stability of Measurement. Finally we discuss the stability of SPPX-STM measurement. A slightly asymmetrical appearance in the $\Delta I$ curve was observed as shown in Figure 4(b), and the values of $\Delta I$ measured in the AlGaAs region are small for $t_{d}=+4.6$ and +11 ps (Figure 6(a)). Since the sample used in the present study was not intentionally doped, the tunneling current hardly flowed and the measurement was carried out with the tip-sample distance being fairly closed. Therefore, as shown in the STM image in Figure 2, the tip was often unstable during the scan and the tunneling current was noisy, which results in instability in SPPXSTM measurement. For example, the noise increases the tipsample distance, reducing the value of $\Delta I$. This instability is considered responsible for the asymmetrical appearance in the $\Delta I$ curve shown in Figure 4(b), and for the smaller values of $\Delta I$ measured in the AlGaAs region for $t_{d}=+4.6$ and $+11 \mathrm{ps}$ (Figure 6(a)).

\section{Conclusions}

We performed SPPX-STM on an LT-GaAs/AlGaAs/GaAs heterostructure. With the AlGaAs layer as a barrier, the ultrashort recombination lifetime of LT-GaAs, $4.0 \mathrm{ps,} \mathrm{was}$ separately obtained from that of GaAs, $4.8 \mathrm{~ns}$. We directly demonstrated the high temporal resolution of SPPX-STM by showing the recombination lifetime of carriers in LT-GaAs $(4.0 \mathrm{ps})$ in the range of subpicosecond temporal resolution. The spatial resolution was discussed in consideration of the screening length of the electric field from the STM probe and the effect of the built-in potential in the sample structure. Since SPPX-STM is a novel microscopy technique and the measured signals are associated with a variety of physical processes, the interpretation of data is not straightforward currently. However, as shown in this paper, careful physical considerations and comparison with results obtained by other techniques provide a better understanding of the physical processes occurring in the measurement. Understanding of the basic mechanisms will enhance the value of this microscopy technique. With the use of selectedwavelength and circularly polarized illumination, quantum transitions including exited and/or spin states are expected to be probed.

\section{Acknowledgments}

This work was supported by a Grant-in-Aid for scientific research from the Ministry of Education, Culture, Sports, Science, and Technology of Japan. The authors thank Professor Y. Hirayama, Tohoku University, for his fruitful discussion.

\section{References}

[1] T. Shinada, S. Okamoto, T. Kobayashi, and I. Ohdomari, "Enhancing semiconductor device performance using ordered dopant arrays," Nature, vol. 437, no. 7062, pp. 1128-1131, 2005.

[2] B. Cheng, S. Roy, G. Roy, F. Adamu-Lema, and A. Asenov, "Impact of intrinsic parameter fluctuations in decanano MOSFETs on yield and functionality of SRAM cells," SolidState Electronics, vol. 49, no. 5, pp. 740-746, 2005.

[3] S. I. Takagi, J. L. Hoyt, J. J. Welser, and J. F. Gibbons, "Comparative study of phonon-limited mobility of two-dimensional electrons in strained and unstrained $\mathrm{Si}$ metal-oxide-semiconductor field-effect transistors," Journal of Applied Physics, vol. 80, no. 3, pp. 1567-1577, 1996.

[4] R. J. Hamers and D. G. Cahill, "Ultrafast time resolution in scanned probe microscopies," Applied Physics Letters, vol. 57, no. 19, pp. 2031-2033, 1990.

[5] G. Nunes Jr. and M. R. Freeman, "Picosecond resolution in scanning tunneling microscopy," Science, vol. 262, no. 5136, pp. 1029-1032, 1993.

[6] G. M. Sleeves, A. Y. Elezzabi, and M. R. Freeman, "Advances in picosecond scanning tunneling microscopy via junction mixing," Applied Physics Letters, vol. 70, no. 14, pp. 1909-1911, 1997.

[7] S. Grafström, P. Schuller, J. Kowalski, and R. Nuemann, "Thermal expansion of scanning tunneling microscopy tips under laser illumination," Journal of Applied Physics, vol. 83, pp. 3453-3460, 1998.

[8] J. Jersch, F. Demming, I. Fedotov, and K. Dickmann, “Timeresolved current response of a nanosecond laser pulse illuminated STM tip," Applied Physics A, vol. 68, no. 6, pp. 637-641, 1999.

[9] V. Gerstner, A. Knoll, W. Pfeiffer, A. Thon, and G. Gerber, "Femtosecond laser assisted scanning tunneling microscopy," Journal of Applied Physics, vol. 88, no. 8, pp. 4851-4859, 2000.

[10] N. N. Khusnatdinov, T. J. Nagle, and G. Nunes, "Ultrafast scanning tunneling microscopy with $1 \mathrm{~nm}$ resolution," Applied Physics Letters, vol. 77, no. 26, pp. 4434-4436, 2000.

[11] S. Loth, M. Etzkorn, C. P. Lutz, D. M. Eigler, and A. J. Heinrich, "Measurement of fast electron spin relaxation times with atomic resolution," Science, vol. 329, no. 5999, pp. 1628-1630, 2010 . 
[12] S. W. Wu and W. Ho, "Two-photon-induced hot-electron transfer to a single molecule in a scanning tunneling microscope," Physical Review B, vol. 82, Article ID 085444, 8 pages, 2010.

[13] Y. Terada, S. Yoshida, O. Takeuchi, and H. Shigekawa, "Realspace imaging of transient carrier dynamics by nanoscale pumpg-probe microscopy," Nature Photonics, vol. 4, no. 12, pp. 869-874, 2010.

[14] O. Takeuchi, R. Morita, M. Yamashita, and H. Shigekawa, "Development of time-resolved scanning tunneling microscopy in femtosecond range," Japanese Journal of Applied Physics, vol. 41, no. 7B, pp. 4994-4997, 2002.

[15] O. Takeuchi, M. Aoyama, R. Oshima et al., "Probing subpicosecond dynamics using pulsed laser combined scanning tunneling microscopy," Applied Physics Letters, vol. 85, no. 15, p. 3268,2004

[16] O. Takeuchi, M. Aoyama, and H. Shigekawa, "Analysis of time-resolved tunnel current signal in sub-picosecond range observed by shaken-pulse-pair-excited scanning tunneling miscroscopy," Japanese Journal of Applied Physics, vol. 44, no. 7B, pp. 5354-5357, 2005.

[17] O. Takeuchi, M. Aoyama, H. Kondo, A. Taninaka, Y. Terada, and H. Shigekawa, "Nonlinear dependences in pulse-pairexcited scanning tunneling microscopy," Japanese Journal of Applied Physics, vol. 45, no. 3B, pp. 1926-1930, 2006.

[18] Y. Terada, M. Aoyama, H. Kondo, A. Taninaka, O. Takeuchi, and H. Shigekawa, "Ultrafast photoinduced carrier dynamics in GaNAs probed using femtosecond time-resolved scanning tunnelling microscopy," Nanotechnology, vol. 18, no. 4, Article ID 044028, 2007.

[19] H. Shigekawa, S. Yoshida, O. Takeuchi et al., "Nanoscale dynamics probed by laser-combined scanning tunneling microscopy," Thin Solid Films, vol. 516, no. 9, pp. 2348-2357, 2008.

[20] Y. Terada, S. Yoshida, O. Takeuchi, and H. Shigekawa, "Laser-combined scanning tunnelling microscopy for probing ultrafast transient dynamics," Journal of Physics: Condensed Matter, vol. 22, no. 26, Article ID 264008, 2010.

[21] H. Shigekawa, O. Takeuchi, Y. Terada, and S. Yoshida, "STM based techniques combined with optics," in Handbook of Nanophysics, K. D. Sattler, Ed., vol. 6 of Principles and Methods, Taylor \& Francis, 2010.

[22] O. Takeuchi, S. Yoshida, and H. Shigekawa, "Light-modulated scanning tunneling spectroscopy for nanoscale imaging of surface photovoltage," Applied Physics Letters, vol. 84, no. 18, pp. 3645-3647, 2004.

[23] S. Yoshida, Y. Kanitani, R. Oshima, Y. Okada, O. Takeuchi, and H. Shigekawa, "Microscopic basis for the mechanism of carrier dynamics in an operating $\mathrm{p}-\mathrm{n}$ junction examined by using light-modulated scanning tunneling spectroscopy," Physical Review Letters, vol. 98, no. 2, Article ID 026802, 2007.

[24] S. Yoshida, Y. Kanitani, O. Takeuchi, and H. Shigekawa, "Probing nanoscale potential modulation by defect-induced gap states on GaAs(110) with light-modulated scanning tunneling spectroscopy," Applied Physics Letters, vol. 92, no. 10, Article ID 102105, 2008.

[25] S. Yoshida, Y. Kanitani, R. Oshima, Y. Okada, O. Takeuchi, and H. Shigekawa, "Nanoscale mapping of built-in potential in GaAs p-n junction using light-modulated scanning tunneling microscopy," Japanese Journal of Applied Physics, vol. 47, no. 7, pp. 6117-6120, 2008.

[26] M. McEllistrem, G. Haase, D. Chen, and R. J. Hamers, "Electrostatic sample-tip interactions in the scanning tunneling microscope," Physical Review Letters, vol. 70, no. 16, pp. 24712474, 1993.

[27] S. Grafström, "Photoassisted scanning tunneling microscopy," Journal of Applied Physics, vol. 91, no. 4, pp. 1717-1753, 2002.

[28] L. Kronik and Y. Shapira, "Surface photovoltage phenomena: theory, experiment, and applications," Surface Science Reports, vol. 37, no. 1, pp. 1-206, 1999.

[29] D. G. Cahill and R. J. Hamers, "Scanning tunneling microscopy of photoexcited carriers at the $\mathrm{Si}(001)$ surface," Journal of Vacuum Science \& Technology B, vol. 9, no. 2, pp. 564-568, 1991.

[30] Ch. Sommerhalter, Th. W. Matthes, J. Boneberg, P. Leiderer, and M. C. Lux-Steiner, "Tunneling spectroscopy on semiconductors with a low surface state density," Journal of Vacuum Science \& Technology B, vol. 15, no. 6, pp. 1876-1883, 1997.

[31] J. Shah, Ultrafast Spectroscopy of Semiconductors and Semiconductor Nanostructures, Springer, Berlin, Germany, 1999.

[32] A. Othonos, "Probing ultrafast carrier and phonon dynamics in semiconductors," Journal of Applied Physics, vol. 83, no. 4, pp. 1789-1830, 1998.

[33] S. Gupta, M. Y. Frankel, J. A. Valdmanis et al., "Subpicosecond carrier lifetime in GaAs grown by molecular beam epitaxy at low temperatures," Applied Physics Letters, vol. 59, no. 25, pp. 3276-3278, 1991.

[34] R. Yano, Y. Hirayama, S. Miyashita, H. Sasabu, N. Uesugi, and S. Uehara, "Pump-probe spectroscopy of low-temperature grown gaAs for carrier lifetime estimation: arsenic pressure dependence of carrier lifetime during MBE crystal growth," Physics Letters A, vol. 289, no. 1-2, pp. 93-98, 2001. 

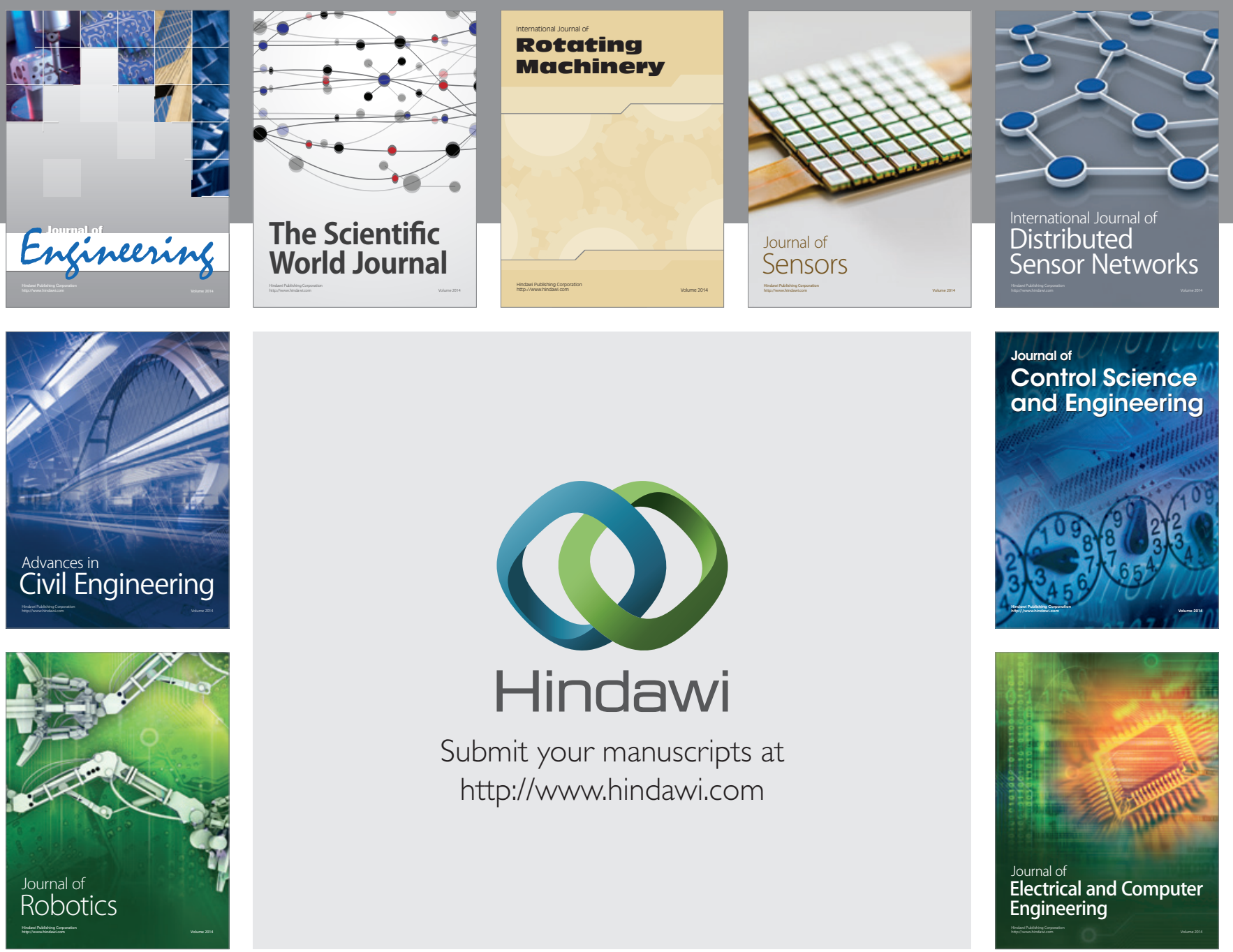

Submit your manuscripts at

http://www.hindawi.com
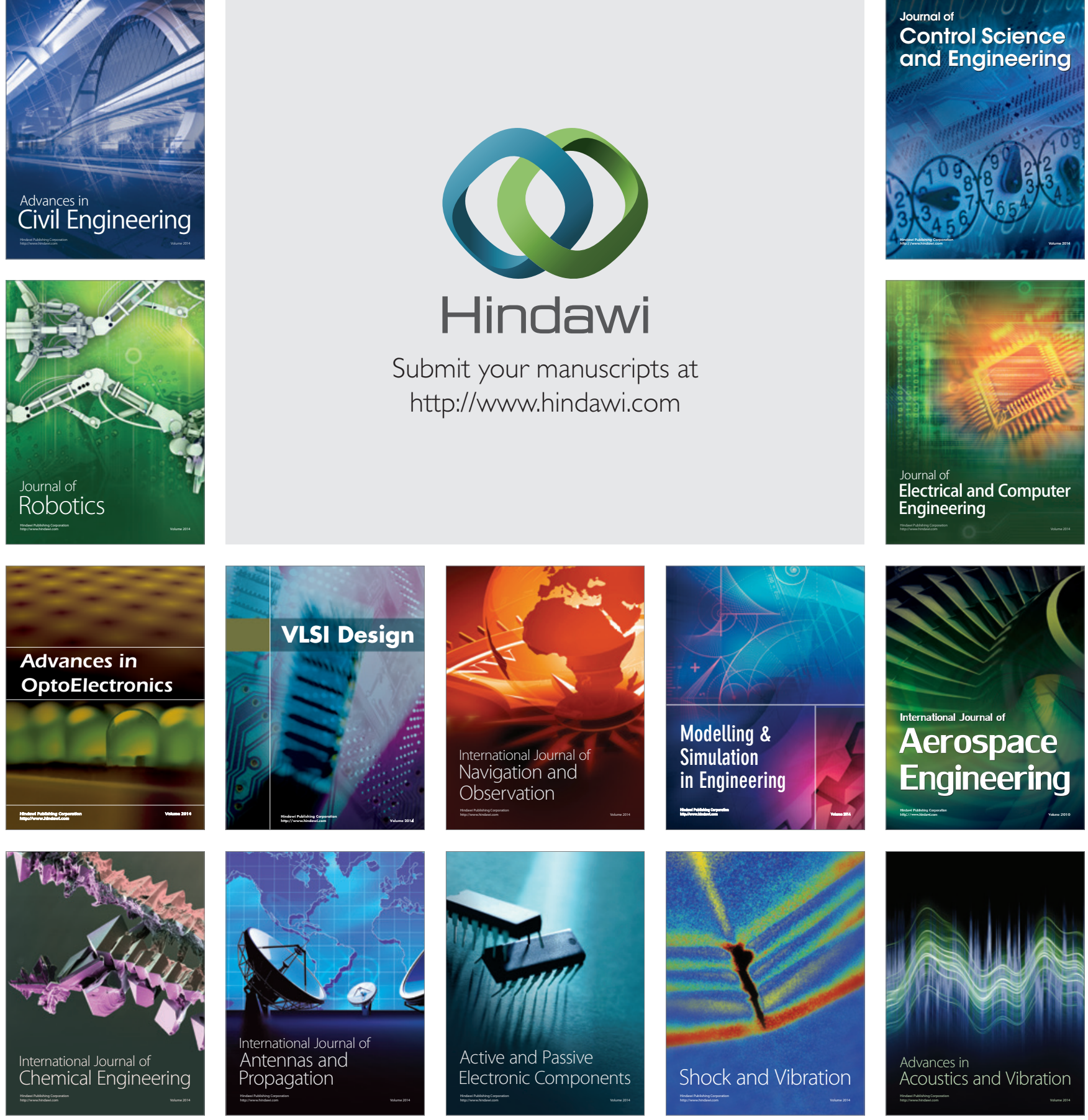